\title{
Influence de l'industrialisation de l'élevage porcin au Vietnam sur la diversification des systèmes en intégration agriculture-élevage
}

\author{
Jean-Daniel Cesaro ${ }^{1,2}$ Vincent Porphyre 1,2 \\ Guillaume Duteurtre ${ }^{1,2}$
}

\section{Mots-clés}

Porcin, polyculture élevage, intensification, développement agricole, Viêt Nam

Submitted: 18 July 2017

Accepted: 22 November 2017

Published: 9 July 2018

DOI : $10.19182 /$ remvt.31277

\section{Résumé}

Depuis le début des années 1990, les systèmes d'élevage porcin au Vietnam ont évolué vers des systèmes plus intensifs qui sont depuis les années 2010 en cours $d^{\prime}$ 'industrialisation. Les éleveurs ont de plus en plus recours à des technologies et des services issus du secteur agro-industriel. Cette transformation des ateliers porcins permet $d^{\prime}$ augmenter la production de viande mais elle remet en cause l'intégration traditionnelle entre l'agriculture et l'élevage au sein des exploitations. A travers une approche historique et géographique, cet article montre que, même dans un processus d'industrialisation du secteur porcin, I'association agriculture élevage perdure. En 2011, plus de 90 \% des exploitations porcines incluaient au moins une activité agricole complémentaire. Cependant la transformation des systèmes d'élevage engendre une diversification des formes d'intégration. Les nouveaux systèmes s'organisent plus autour de la gestion des effluents pour développer des ateliers agricoles à plus forte valeur ajoutée, ou sur la complémentarité économique entre plusieurs spéculations face aux incertitudes de marchés, et de moins en moins vers la production sur l'exploitation de fourrages ou d'aliments bétail.

- Comment citer cet article : Cesaro J.-D., Porphyre V., Duteurtre G., 2018. Influence of the industrialization of pig farming on the diversification of mixed livestock integration in Vietnam. Rev. Elev. Med. Vet. Pays Trop., 71 (1-2): 7-13, doi: 10.19182/remvt.31277

\section{INTRODUCTION}

L'élevage porcin fournit $74 \%$ de la viande produite au Vietnam et $64 \%$ de la viande consommée dans le pays (FAOstat, 2017). Depuis les années 1980, la demande en viande augmente du fait de la croissance démographique et de l'élévation du niveau de vie. Entre 1980 et 2016, la population est passée de 54 à 92 millions d'habitants, soit une augmentation de $71 \%$ (FAOstat, 2017). Le produit intérieur brut par habitant est passé de 100 à 2200 USD par an (World Bank, 2016). En 2014, les achats de viande de porc représentaient environ $15 \%$ des dépenses alimentaires des ménages (Nguyen Tien Dinh, 2014). C'est donc un secteur agricole stratégique pour la souveraineté alimentaire du pays. Entre 1990 et 2015, la production porcine a été multipliée par six, passant de 0,53 à plus de 3,3 millions de tonnes en trente ans (FAOstat, 2017), alors que le cheptel porcin est passé de 11 à près de 27 millions de têtes (GSO, 2016).

1. CIRAD, UMR SELMET, F-34398 Montpellier, France.

2. SELMET, Univ Montpellier, CIRAD, INRA, Montpellier SupAgro, Montpellier, France.
Cette augmentation de production et de productivité en élevage porcin a été pour l'essentiel le fait des exploitations familiales (Lapar et Staal, 2010). Suite aux réformes économiques des années 1980, de nombreux ménages ruraux et périurbains s'engagent dans l'élevage intensif (Molénat et Tran The Thong, 1991). Cette « intensification» des systèmes d'élevage correspond à un processus d'industrialisation des filières, au sens où les éleveurs utilisent des technologies, des intrants et des services issus des agro-industries (Gura, 2008). Avec le développement des usines d'aliment pour bétail, les éleveurs ont de plus en plus recours à l'aliment concentré, délaissant les sous-produits agricoles disponibles localement (Martin et al., 2004 ; Dao The Anh, et al., 2005 ; Porphyre et Nguyen Que Coi, 2006). L'élevage porcin domestique traditionnel commence à perdre de son importance aussi bien en terme de production que de nombre d'exploitations. La géographie des systèmes d'élevage se met alors à changer (Epprecht, 2005), générant une large palette de modèles d'exploitations (Hanh et al., 2017).

Cette évolution soulève de nombreuses questions liées au développement durable de ces productions intensives (Lemke et Valle Zárate, 2008 ; Duteurtre et Vu Trong Binh, 2010). Parmi les enjeux sensibles liés à l'essor actuel de la production porcine au Vietnam, figurent la 
gestion des effluents d'élevage dans les zones périurbaines, les risques de pollution des eaux, et la promotion des complémentarités entre activités agricoles et d'élevage à l'échelle des territoires (Porphyre et Nguyen Que Coi, 2006 ; Lapar, 2012).

Dans la littérature internationale (Seré et Steinfeld, 1995 ; Naylor et al., 2005 ; Robinson et al., 2011), l'industrialisation des systèmes d'élevage est décrite comme un remplacement d'une production fondée sur l'utilisation de la terre par des ateliers hors-sol utilisant des intrants agro-industriels. Cette perte de lien entre les productions animales et la ressource agricole locale est définie comme un « découplage » systémique et géographique entre des activités a priori nécessaires pour le bon fonctionnement d'une exploitation durable (Naylor et al., 2005 ; Moraine, 2015). Au Vietnam, le découplage progressif entre production agricole et système d'élevage n'intervient pas de façon homogène sur l'ensemble du territoire. Si l'industrialisation se vérifie à l'échelle nationale, localement, les systèmes semblent se transformer à des degrés divers, ce qui remet en cause l'hypothèse d'une généralisation des systèmes hors-sol. Malheureusement, il existe très peu de données disponibles permettant d'appréhender le degré d'intégration agriculture-élevage au niveau des exploitations et des territoires. Afin de mieux anticiper les risques liés à l'intensification des élevages porcins, l'objectif du présent article a été d'analyser l'évolution des liens entre l'élevage et les cultures au sein des exploitations porcines au Vietnam. Il s'agissait aussi de décrire la diversité géographique de la production porcine et ainsi de montrer que le processus national d'industrialisation de l'élevage est d'abord l'expression de multiples dynamiques locales.

\section{MATERIEL ET METHODES}

\section{Données issues des recensements agricoles}

L'étude a considéré les exploitations d'élevage dans leur diversité et dans leur globalité, c'est-à-dire en considérant l'atelier d'élevage porcin inséré dans un système plus large d'activités agricoles. Afin de mieux connaitre la dynamique de transformation de ces exploitations, l'étude s'est attachée à décrire leur évolution à la fois dans le temps (approche historique) et dans l'espace (approche géographique). Les données historiques proviennent d'une analyse de la bibliographie sur la question. Les données quantitatives soutenant l'approche géographique proviennent des recensements agricoles du General Statistics Office du Vietnam (GSO, 1994 ; 2001 ; 2006 ; 2011), ainsi que les données statistiques agricoles sectorielles de 2015 (GSO, 2016). Ces données ont été complétées par une revue de la littérature sur le contexte historique, sociopolitique, environnemental et économique de l'élevage porcin au Vietnam, ainsi que par une analyse des zones agroécologiques du pays.

\section{Mobilisation de la typologie internationale des systèmes d'élevage}

Pour décrire la diversité des systèmes d’élevage, il convient de prendre en compte la question de l'alimentation, des associations culturales, de l'utilisation des sous-produits agricoles et des modes de gestion des effluents, c'est-à-dire des modes d'intégration agriculture-élevage. Dans la typologie internationale des systèmes d'élevage, Seré et Steinfeld (1995) différencient deux grands types de systèmes d'élevage : les systèmes spécialisés hors-sol (aliment pour bétail acheté hors ferme) et les systèmes en polyculture-élevage (en culture irriguée ou pluviale).

Avec les données disponibles dans les recensements agricoles vietnamiens (GSO, 2001 ; 2006 ; 2011), il n'est pas possible de connaître l'origine de l'aliment dans un système d'élevage puisque les données sont structurelles (superficie agricole, nombre de têtes, etc.). De même, il n'est pas possible de différencier les cultures irriguées des pluviales. Il n'est donc pas possible de reproduire exactement la typologie internationale des systèmes d'élevage de Seré et Steinfeld (1995) avec les données des recensements agricoles. Il est cependant possible de s'en approcher.

Pour chaque exploitation, nous connaissions le nombre d'animaux et la superficie agricole par type de culture (annuelle, pérenne, aquaculture, forêt). Il était donc possible de distinguer les exploitations porcines avec au moins des cultures annuelles (intégration potentielle), avec des cultures pérennes, de l'aquaculture ou des espaces boisés (système en polyculture-élevage [mixed systems ou landbased systems]) et enfin celles sans terre (hors-sol [landless systems]).

Pour différencier les systèmes faiblement intensifs des systèmes intensifs, la typologie internationale de Seré et Steinfeld (1995) utilise le seuil de 10 unités d'élevage (UE). Les UE sont des facteurs de conversion d'un animal en une unité permettant le calcul d'un stock animal total (Eurostat, 2017). Dans les systèmes potentiellement mixtes, nous avons distingué les élevages selon leur potentiel d'intégration agriculture-élevage en trois classes définies par le nombre d'UE : les élevages avec moins de dix UE à fort potentiel d'intégration (S1), les élevages entre dix et vingt UE ayant un potentiel intermédiaire (S2), enfin les élevages avec plus de vingt UE au fort potentiel de découplage $(\mathrm{S} 3)$. Pour les systèmes avec des cultures non-annuelles, nous avons distingué les systèmes avec moins de dix UE (faiblement intensif) (S4) des systèmes avec plus de dix UE (intensif) (S5). Pour finir, les systèmes hors-sol ont été classés dans la même catégorie (S6) (figure 1).

\section{RESULTATS}

\section{Grande transition de l'élevage porcin}

\section{Sortie du modèle collectiviste et marchandisation de l'élevage porcin (1986-1993)}

Pendant la période collectiviste, l'élevage porcin était officiellement limité aux coopératives (hợp tác xã) et fermes d'Etat (nông trường) (Dao The Tuan, 2002). L'élevage porcin marchand familial était interdit puisqu'il favorisait la capitalisation au sein de ménage (Tran The Tong, 1973). Au-delà des postures idéologiques, cette activité, dite « complémentaire » à l'économie collectivisée, était tolérée par les autorités locales car le marché noir était lucratif.

Avec les réformes dites du Renouveau (Đổi mói i) de décembre 1986, l'agriculture a été progressivement décollectivisée et le marché noir alimentaire s'est progressivement légalisé sans pour autant se formaliser. Les paysans vietnamiens ont utilisé l'élevage porcin pour sortir de la pauvreté économique (Molénat et Tran The Thong, 1991). La demande en viande a été dynamique et le marché intérieur protégé. L'élevage porcin permet de capitaliser les revenus des ménages ruraux. Ces derniers se concentrent sur l'élevage de truies et de porcelets. Les éleveurs se détournent de l'engraissement car les ressources agricoles restent limitées. Selon le recensement agricole de 1994, $99 \%$ des exploitations ont moins de dix porcs (GSO, 1994).

\section{Intensification des systèmes d'élevage par une révolution verte tardive (1993-2008)}

Au milieu des années 1990, les productions agricoles commencent à s'intensifier. L'ouverture économique du pays améliore l'accès aux semences hybrides et aux engrais chimiques. En 1993, la réforme foncière donne des droits d'usage aux ménages ruraux pour cultiver les terres des anciennes coopératives. Ces conditions favorisent une augmentation de rendements. Le secteur des cultures ne se limite plus seulement au riz et se diversifie avec le développement du maïs et du manioc. Un marché de matières premières agricoles se met en 
Typologie des systèmes d'élevage adaptée aux données des recensements agricoles du Vietnam

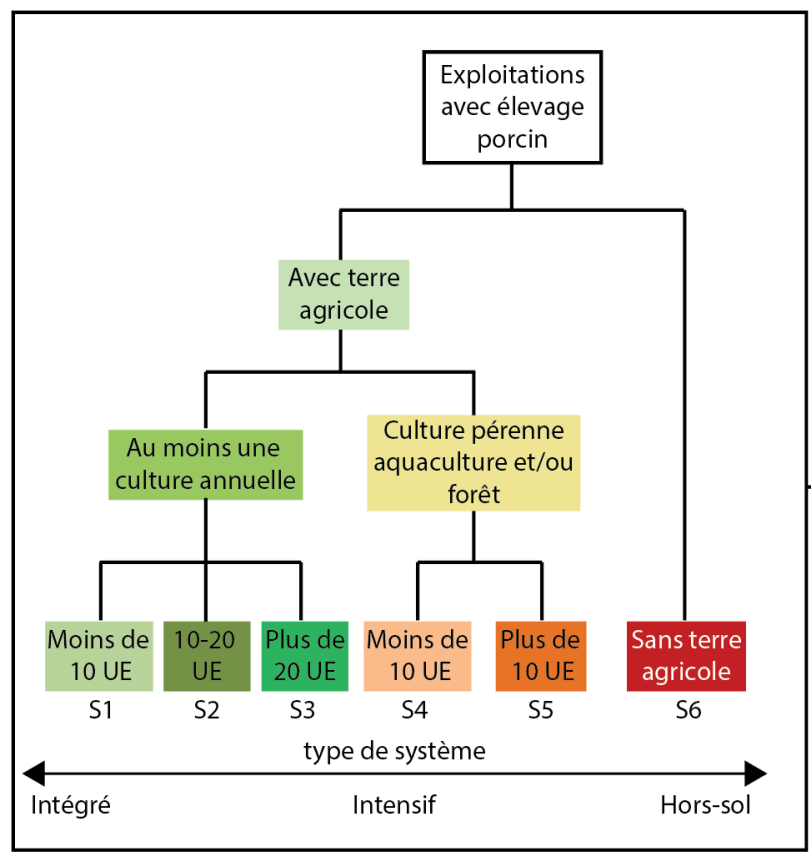

Extraction des données du recensements agricoles du Vietnam et application de la typologie

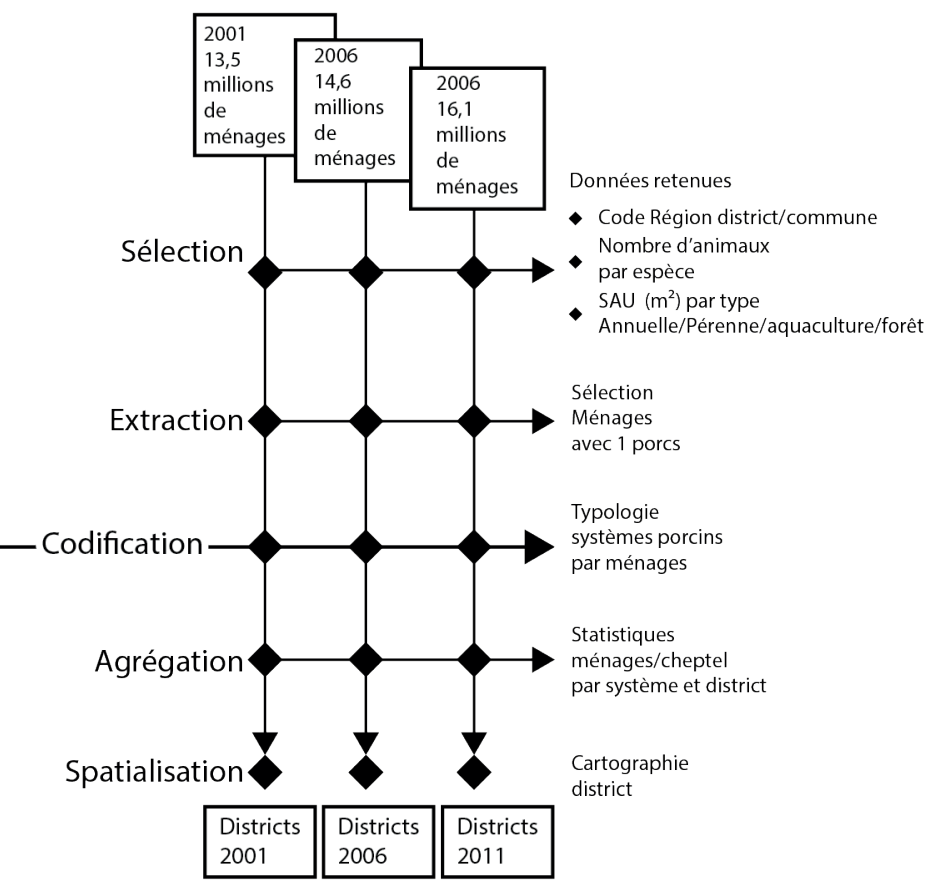

Figure 1 : typologie des systèmes d'élevage porcins au Vietnam et protocole de classification dans les systèmes de gestion des bases de données des recensements agricoles et ruraux de 2001, 2006 et 2011. UE : unité d'élevage ; SAU: surface agricole utile.

place dans le pays. Avec l'essor de la demande urbaine en viande, les paysans commencent à engraisser leurs animaux (Pingali, 1997). Les ménages situés à proximité des marchés urbains se spécialisent dans l'activité de finisseur (Vu Trong Binh, 2002). Une spécialisation géographique apparaît entre les naisseurs, les engraisseurs et les finisseurs avec l'organisation de filières informelles et la construction socioéconomique du prix (Goulven et al., 1999). En 2001, le secteur compte huit millions d'exploitations porcines. Selon le recensement, $1 \%$ des exploitations ont plus de dix porcs et produisent $10 \%$ de la production.

Au début des années 2000, le gouvernement cherche à promouvoir une intensification des exploitations porcines. Il soutient alors un modèle d'exploitation appelé « jardin, aquaculture, élevage » (virơnn ao chuồng ou VAC) (Porphyre et Nguyen Que Coi, 2006 ; Gironde, 2008). Cette forme d'agriculture valorise une boucle fermée de nutriments. Le fumier des porcs sert à nourrir les poissons. L'eau des bassins est ensuite utilisée pour irriguer les cultures. Les résidus de cultures et le son de riz sont utilisés dans l'alimentation des porcs. Avec la réforme agraire de 2003, l'Etat privatise quelques terres de rizières aux abords des villages pour créer des ateliers porcins et des bassins piscicoles à grande échelle (Mikolasek et al., 2009).

Parallèlement, les agro-industries continuent de s'implanter dans le pays. Selon Smith (2017), le ministère de l'Agriculture américain (USDA) estime en 2000 que $20 \%$ des aliments utilisés en élevage sont transformés par des agro-industries. Au début, seules les exploitations intensives, comme les VAC, sont capables d'acheter ces produits. Derrière un modèle théorique du VAC se trouve une réalité plus linéaire où la production porcine est progressivement industrialisée (Gironde, 2008). Les effluents servent à intensifier les systèmes agricoles environnants. Les cultures annuelles, notamment le riz et le maïs, sont encore un peu cultivées pour la nutrition animale mais les cultures pérennes se développent rapidement dans le sillon des exploitations intensives (Van Thi Khanh Vu, Tien Tran Minh et Son Thi Thanh Dang, 2007). Entre 2000 et 2005, le renchérissement des prix agricoles freine la rentabilité de l'élevage porcin. En 2006, environ sept millions d'exploitations continuent d'élever des porcs, soit un million de moins qu'en 2001. La concentration amorcée par les VAC pousse la restructuration du marché soutenue par l'Etat et les agro-industries. Le nombre de porcs par exploitation passe de 1,25 à 2,5. En 2006, $5 \%$ des exploitations détiennent plus de dix porcs et représentent $35 \%$ de la production.

Restructuration et émergence des fermes commerciales (2008-2016)

En 2007, le Vietnam intègre l'Organisation mondiale du commerce. Son marché s'ouvre à la concurrence. L'activité d'engraissement devient de plus en plus compétitive, surtout dans un contexte de concurrence accrue. La dévaluation du dong face au dollar impacte directement les éleveurs de plus en plus dépendants des importations agricoles, notamment pour le soja. La crise des matières agricoles en 2007-2008 plonge le secteur porcin dans une crise grave (Fulton et Reynolds, 2015). Selon le dernier recensement agricole, le nombre d'exploitations avec un élevage porcin atteint 4,2 millions en 2011. Environ $10 \%$ des exploitations détiennent plus de dix porcs et produisent plus de $50 \%$ de la production nationale (GSO, 2011).

Comme le montre la figure 2, la restructuration a été en faveur des exploitations de tailles moyennes (entre dix et cinquante porcs), cette catégorie captant plus de $35 \%$ de la production en 2011 contre moins de $10 \%$ en 2001. En nombre d'exploitations, cette catégorie augmente alors même que le nombre de petits éleveurs diminue fortement. Les grandes exploitations restent marginales numériquement, représentant moins de $0,1 \%$ des exploitations du pays, mais comptent pour plus de $15 \%$ de la production en 2011. L'intensification et l'industrialisation ont donc eu pour effet de faire émerger un nombre élevé d'exploitations de taille moyenne et un nombre plus faible de grandes exploitations.

En 2008, le gouvernement publie la stratégie de l'élevage à l'horizon 2020. Cette stratégie affiche l'ambition d'orienter le secteur de l'élevage vers une production plus intensive et industrielle. Pour soutenir 
Nombre d'exploitations avec des porcs selon le niveau de production entre 2001 et 2011

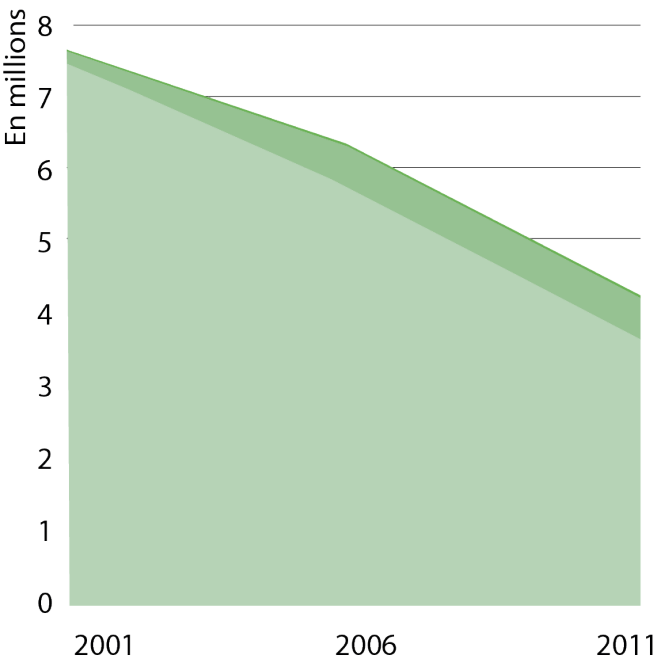

Cheptel porcin (têtes)

par niveau de production entre 2001 et 2011

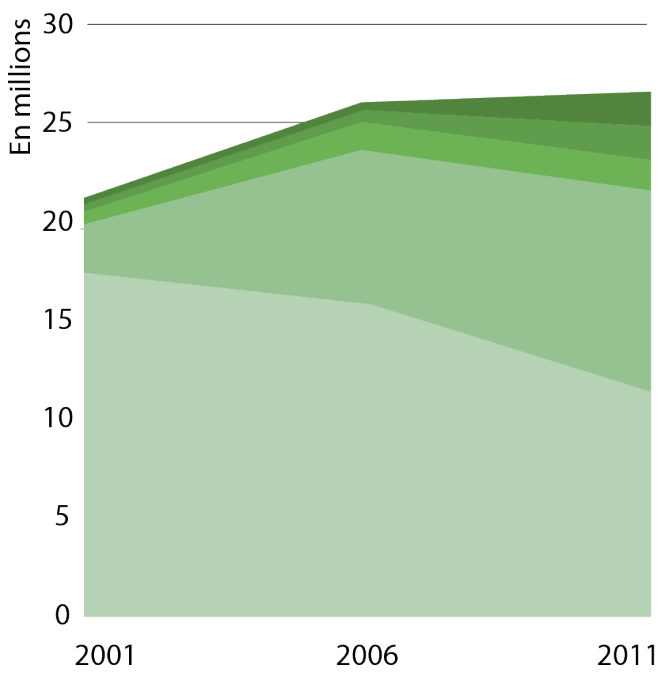

Niveau de production (Nombre de porcs par exploitation)

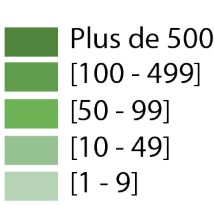

Calculs des auteurs à partir des données RGA GSO, 2001; $2006 ; 2011$

Figure 2 : restructuration du secteur porcin au Vietnam entre 2001 et 2011.

cette transformation, le gouvernement crée en 2011 une catégorie d'exploitations agricoles modèle pour moderniser le secteur agricole. Ces exploitations sont appelées littéralement « ferme » (trang trai que nous traduisons par « fermes commerciales ») (Kojin, 2013). Pour devenir une ferme commerciale, une exploitation doit réaliser un chiffre d'affaire d'un milliard de dongs (50 000 USD en 2011). Selon les fluctuations du marché, il faut entre 250 et 400 porcs par exploitation pour obtenir le statut de ferme commerciale. Entre 2011 et 2015, le nombre de fermes commerciales d'élevage a plus que doublé, passant de 6267 à 15068 (GSO, 2016). La majorité d'entre elles sont spécialisées dans la production porcine et avicole. Selon les données du recensement, ces fermes représentaient environ $5 \%$ de la production porcine nationale en 2011 et leur contribution atteindrait $20 \%$ de la production en 2016 (résultat provisoire, GSO, 2016).

Entre 2006 et 2017, cette restructuration du secteur a permis de produire deux fois plus de viande sans augmenter le cheptel national. Les indicateurs de productivité se sont nettement améliorés : nombre de porcelets par truie, nombre de porcelets engraissés, poids moyen des porcs charcutiers en fin de cycle (Nguyen Manh Dzung, 2014). Le développement du marché de l'aliment industriel a permis cette modernisation du secteur. En 2015, l'USDA estime que la moitié de l'aliment des exploitations d'élevage au Vietnam sont issus de l'industrie (Smith, 2017). Le pays importe douze millions de tonnes de matières premières agricoles pour la nutrition animale (FAOstat, 2017). Les systèmes d'élevage porcin sont donc passés d'une organisation autonome, peu productive, donc peu marchande, à un secteur industrialisé intensif, fortement orienté vers le commerce, et dépendant des importations agricoles. Dans quelle mesure cette restructuration et cette industrialisation entrainent-elles une transformation des systèmes d'élevage?

\section{Dynamique des systèmes d'élevage au prisme de l'intégration agriculture-élevage au sein des exploitations}

\section{Une production porcine encore bien intégrée à l'agriculture locale}

En affectant notre typologie des systèmes d'élevage (figure 1) aux données des trois recensements agricoles de 2001, 2006 et 2011, nous pouvons analyser l'évolution des six systèmes d'élevage en termes de nombre d'exploitations et de nombre de porcs (figure 3). En 2001, les systèmes à fort potentiel d'intégration étaient dominants pour ces critères (nombre d'exploitations et d'animaux) à l'échelle nationale. Cette catégorie a néanmoins diminué au cours de la décennie, suite à la sortie du secteur des petits engraisseurs. Les systèmes à faible potentiel d'intégration se sont développés entre 2006 et 2011 pour représenter aujourd'hui plus de la moitié de la production par seulement moins de $10 \%$ des exploitations. Dans le même temps, les systèmes hors-sol mixtes (avec culture pérenne et aquaculture) atteignent presque $15 \%$ de la production alors qu'ils ne représentent même pas $1 \%$ des exploitations. Les systèmes hors-sol spécialisés, les vrais « sans-terre », représentent $7 \%$ de la production. Ce sont majoritairement des éleveurs périurbains encerclés par les fronts de périurbanisation et de très grandes fermes spécialisées appartenant à des firmes, situées dans des régions relativement excentrées pour des raisons de biosécurité.

\section{Divergence des systèmes de production entre le nord et le sud du Vietnam}

En agglomérant la contribution de chaque système d'élevage au cheptel du district, il est possible de réaliser une typologie des systèmes d'élevage en suivant une classification ascendante hiérarchique (CAH) en 2001, 2006 et 2011 (figure 4). Les résultats montrent des formes de spécialisation régionale qui s'expliquent par différents facteurs démographiques, économiques et géographiques. Les systèmes hors-sol mixtes (S4, S5) et hors-sol spécialisés (S6) sont surreprésentés autour de Ho Chi Minh-Ville, notamment dans la partie nord de la région métropolitaine dans les zones de cultures pérennes (caoutchouc, café). Les systèmes d'élevage porcin du delta du Mékong restent en majorité peu intensifs, sauf autour de la ville de Ben Tre et le long de la route en direction du Cambodge. Dans le nord du pays, les systèmes à faible potentiel d'intégration (S2 et S3) se sont développés, d'abord autour de la route de Hai Phong à Hanoi, puis dans le reste du delta du fleuve Rouge. A l'inverse, les régions d'altitude, littorales et les régions rizicoles du delta du Mékong conservent une surreprésentation de système d'élevage porcin à fort potentiel d'intégration (S1).

Dans la région du delta du fleuve Rouge, la dynamique dominante tend vers une intensification générale des systèmes mixtes ( $\mathrm{S} 1$ vers $\mathrm{S} 3$ ). Une seconde transition apparaît plus localement avec un développement de systèmes porcins hors-sol couplés à des cultures pérennes et des bassins aquacoles (S4 et S5). Ces nouveaux systèmes apparaissent de manière privilégiée le long des berges fluviales et des grands canaux pour des raisons d'accès à l'eau. Ce phénomène est en lien avec le développement des exploitations associant l'aquaculture et les cultures 
pérennes. Dans la périphérie d'Ho Chi Minh-Ville, la phase d'industrialisation semble au contraire porter une nouvelle réintégration des systèmes hors-sol avec des cultures pérennes (S2, S6 vers S4, S5). Les systèmes hors-sol spécialisés (S6) en périphérie des métropoles sont voués à disparaître. Dans le reste du pays, l'intensification à l'œuvre se mélange avec des formes moins intensives mais beaucoup plus intégrées aux cultures locales (S1 vers S2). La concentration animale dans ces régions dépend de l'intensité des productions agricoles, donc des résidus de cultures disponibles pour l'élevage porcin. Le développement de systèmes intensifs (S3) reste anecdotique dans ces zones.
Nombre d'exploitations avec des porcs selon le type de système entre 2001 et 2011

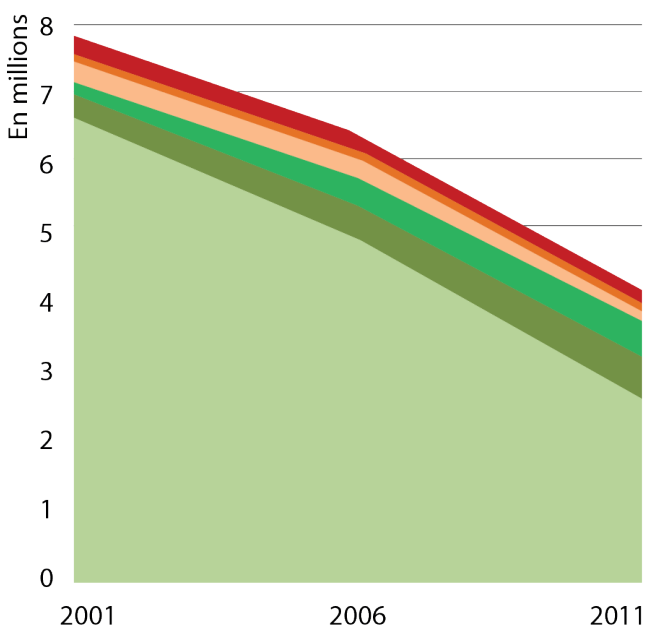

Cheptel porcin (têtes) selon le type de système entre 2001 et 2011

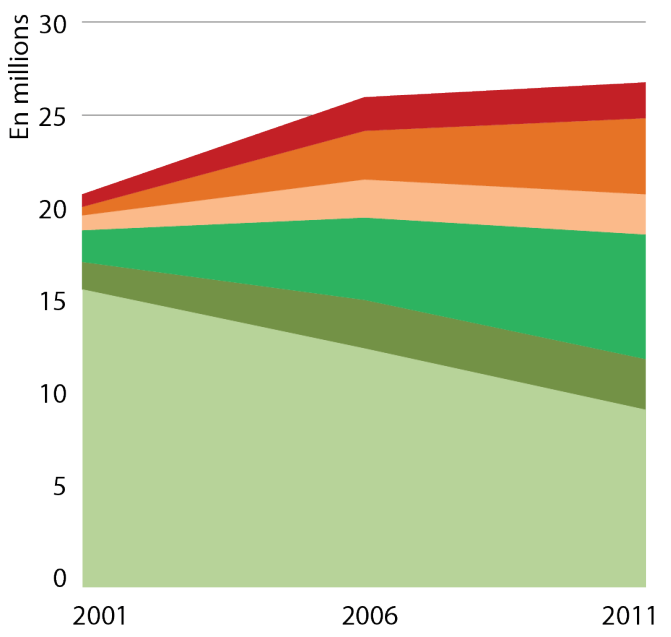

Type de système

Elevage porcin et au moins une culture annuelle
S1 Faible intensité
$\mathrm{S2}$ Moyenne intensité
S3 Forte intensité

Elevage porcin avec au moins une culture pérenne, forestière ou aquacole S4 Faible intensité S5 Forte intensité Elevage porcin sans terre S6 Forte intensité

Calculs des auteurs à partir des données RGA GSO, $2001 ; 2006 ; 2011$

Figure 3 : dynamique des systèmes d'élevage porcin au Vietnam entre 2001 et 2011.
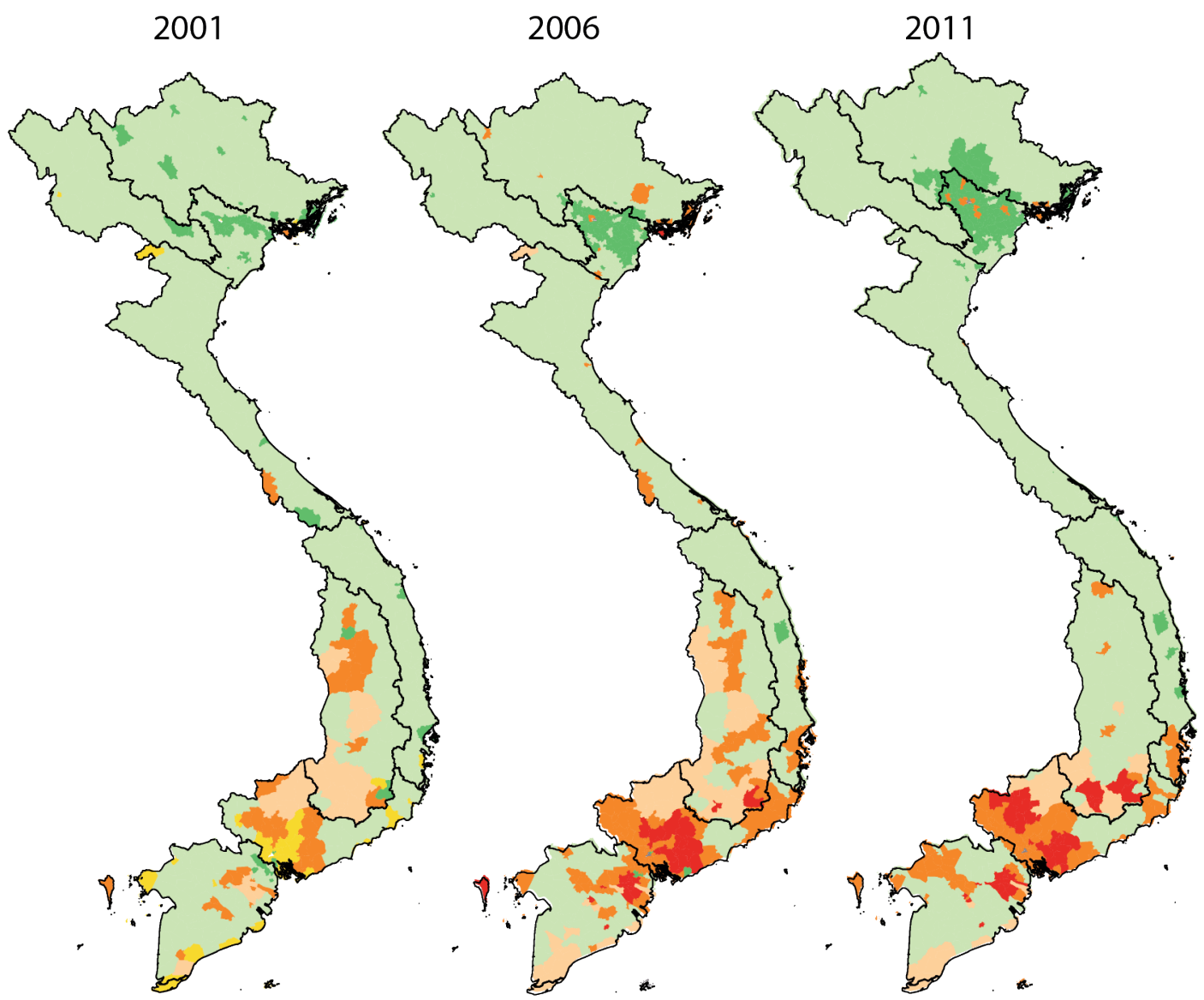

Systèmes porcins dominants par district

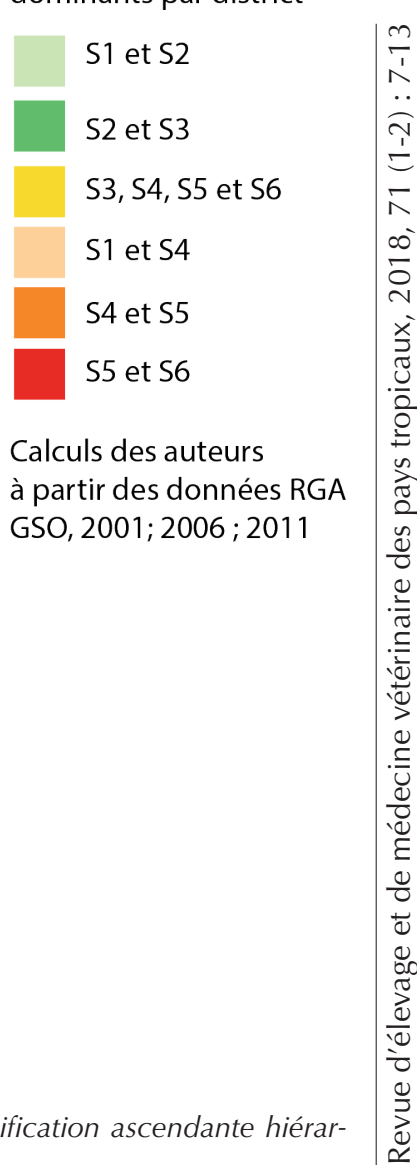

Figure 4 : typologie spatiale de la contribution
chique) en 2001,2006 et 2011 au Vietnam. 


\section{DISCUSSION}

L'analyse de la dynamique des systèmes d'élevage au Vietnam montre une limite en termes de caractérisation de la diversité. En effet, retenir six catégories limite forcément la portée de l'analyse. Il serait intéressant de différencier les systèmes selon qu'ils utilisent strictement des cultures annuelles ou qu'ils associent des cultures annuelles et pérennes. Nous pourrions aller plus loin en mettant en avant le nombre exact de systèmes associant élevage porcin et aquaculture (modèle VAC). Il serait même possible de déterminer un indicateur de diversité culturale au sein des exploitations. Cette première analyse a eu pour but de montrer que les systèmes intensifs n'étaient pas nécessairement des systèmes hors-sol spécialisés, même si l'aliment du bétail provenait d'agro-industries.

Concernant, l'association élevage-agriculture par transfert de fertilité, il serait intéressant de caractériser les besoins en terme de fertilisant et de les comparer avec la concentration de matières organiques d'origine animale par exploitation pour définir la nature intensive des systèmes. Les unités d'élevage restent assez peu explicites en termes de dimensionnement des exploitations rapportées à la surface agricole. Enfin, l'analyse du secteur porcin par système d'élevage fait émerger les trois grandes questions ci-après à propos de la diversité des modèles de production au Vietnam.

\section{Compétition ou complémentarité entre systèmes?}

Sont-ils complémentaires ou voués à s'affronter sur un même marché ? Quels sont les niveaux d'interaction économique, sociale ou environnementale entre les différents systèmes ? Toute une littérature émerge sur ces questions de coexistence et de confrontation (Galliano et al., 2017). Le secteur porcin au Vietnam est un objet d'étude intéressant pour développer ces thématiques de recherche (Diep Thanh Tung, 2016).

\section{Quelle reconnaissance politique?}

Depuis dix ans, le ministère de l'Agriculture du Vietnam insiste sur la modernisation et l'industrialisation du secteur de l'élevage. Le modèle dominant de toute politique demeure l'exploitation commerciale horssol spécialisée à grande échelle (Otsuka et al., 2016). Seulement, la réalité du terrain ne cadre pas avec les visions stratégiques à l'horizon 2020. Comment dès lors amener le ministère de l'Agriculture à accepter la diversité locale dans un souci de modernisation du secteur et de maintien de l'emploi en zone rurale ?

\section{Quelle intégration territoriale des systèmes intensifs?}

Les nouvelles exploitations hors-sol profitent d'une rente de situation alors que les exploitations traditionnelles mixtes, parfois désignées comme « intégrées », privilégient les ressources locales (Moraine, 2015). Evidemment, les expressions « hors-sol » et « intégré » opposent des conceptions linéaires et circulaires de flux de matières à l'échelle d'une exploitation. Mais que faire quand les systèmes s'intensifient ? Faut-il privilégier « un recouplage » systémique ou territorial ? A quelle échelle doit s'organiser le traitement des flux de matières ?

\section{- CONCLUSION}

Les systèmes d'élevage porcin se sont profondément transformés au Vietnam en l'espace de trente ans et les dynamiques actuelles vont se poursuivre, voire s'intensifier, dans un contexte d'ouverture économique toujours plus prononcée. Les élevages se sont intensifiés puis industrialisés dans les régions fortement connectées aux infrastructures de transport maritime et routier, dans une zone d'influence des grandes métropoles que sont Hanoi et Ho Chi Minh-Ville. La concentration de la production animale dans un nombre plus réduit d'exploitations et de fermes commerciales a généré de nouvelles formes d'intégration agriculture-élevage à l'échelle des exploitations. Les élevages porcins intensifs et industrialisés ne sont pas des systèmes complètement hors-sol mais sont bien intégrés à des cultures pérennes ou à de l'aquaculture afin de mieux gérer les effluents. L'intensification et l'industrialisation de l'élevage porcin entraîne une intensification agroécologique au sein des exploitations. Au-delà de ces espaces où les systèmes s'intensifient rapidement, les systèmes porcins traditionnels semblent se maintenir. Dans les régions les plus reculées où les taux de pauvreté sont les plus forts, les ménages ruraux continueront de conduire des petits cheptels porcins pour assurer leurs moyens d'existence. Cependant, ces petits systèmes intégrés coexisteront au niveau national avec des fermes commerciales spécialisées et complètement industrialisées dont la productivité égale, voire dépasse celle d'exploitations des pays développés.

\section{REFERENCES}

Dao The Anh, Pham Thi Hanh Tho, Vu Trong Binh et al., 2005. Evolution of integrated crop-animal systems in Northern Vietnam. In: Integrated crop-animal systems in Southeast-Asia: current status and prospects (Eds Sombilla MA., Hardy B.). IRRI, Los Baños, Philippines, 63-89

Dao The Tuan, 2002. Vietnam. Réformes agraires successives et succès de I'agriculture familiale. In : Forum social mondial, Porto Alegre, Brésil, janvier 2001

Diep Thanh Tung, 2016. Measuring the technical efficiency of livestock production in Vietnam. Outlook Agric., 45 (2): 132-139, doi: 10.1177/0030727016650771

Duteurtre G., Vu Trong Binh, Eds., 2010. In : Proc. Workshop Future prospects for livestock in Vietnam: How to balance livestock industrialization, rural development strategy and environmental changes? Hanoi, 28 Nov. 2010. CIRAD, Montpellier, France / IPSARD, Hanoi, Vietnam, $31 \mathrm{p}$.

Epprecht M., 2005. Geographic dimensions of livestock holdings in Vietnam - Spatial relationships among poverty, infrastructure and the environment. PPLPI/FAO, Italy, Rome, 51 p.

FAOstat, 2017. www.fao.org/faostat/en/ (accessed 3 Apr. 2018)

Fulton M.E., Reynolds T., 2015. The political economy of food price volatility: The case of Vietnam and rice. Am. J. Agric. Econ., 97 (4): 1206-1226, doi: 10.1093/ajae/aav019

Galliano D., Lallau B., Touzard J.-M., 2017. Coexistences et transitions dans I'agriculture. Rev. Fr. Socio-Econ. (18) : 23-30, doi : 10.3917/rfse.018.0023

Gironde C., 2008. Grandes réformes et petits arrangements dans les campagnes vietnamiennes. Autrepart, 48 (4) : 113-127, doi: 10.3917/ autr.048.0113

GSO, 1994. Résultats du recensement agricole du Vietnam de 1994, Vol. 2, GSO, $450 \mathrm{p}$.

GSO, 2001. Résultats du recensement agricole du Vietnam de 200, www. gso.gov.vn/default_en.aspx?tabid=477\&idmid=\&ItemID=1824 (consulté le 3 avr. 2018)

GSO, 2006. Résultats du recensement agricole du Vietnam de 2006, www. gso.gov.vn/default_en.aspx?tabid=477\&idmid=\&ltemID=8074 (consulté le 3 avr. 2018)

GSO, 2011. Résultats du recensement agricole du Vietnam de 2011, www. gso.gov.vn/default_en.aspx?tabid=477\&ltemID=13399 (consulté le 3 avr. 2018)

GSO, 2016. General Statistic Office, www.gso.gov.vn/ (accessed 12 Apr. 2018)

Gura S., 2008. Industrial livestock production and its impact on smallholders in developing countries. League for Pastoral Peoples and Endogenous Livestock Development, Ober-Ramstadt, Allemagne, 65 p. 
Han Quang Hanh, Azadi H., Dogot T., Vu Dinh Ton., Lebailly P., 2017. Dynamics of agrarian systems and land use change in North Vietnam. Land Degrad. Dev., 28 (3): 799-810, doi: 10.1002/ldr.2609

Kojin E., 2013. The development of private farms in Vietnam. Institute of Developing Economies, Japan External Trade Organization (Discussion Paper No. 408

Lapar L., Staal S., 2010. Competitiveness of smallholder pig producers in Vietnam. Improving the competitiveness of pig producers. ILRI, Nairobi, Kenya

Le Goulven K.L., Boutonnet J.-P., Codron J.-M., 1999. Marketing an agricultural production in a "transition" economy: pork marketing chain from Nam Thanh to Hai Phong (Vietnam). Rev. Elev. Med. Vet. Pays Trop., 52 (3-4): 305-312, doi: 10.19182/remvt.9679

Lemke U., Valle Zárate A., 2008. Dynamics and developmental trends of smallholder pig production systems in North Vietnam. Agric. Syst., 96 (13): 207-223, doi: 10.1016/j.agsy.2007.08.003

Martin C., Castella J.-C., Anh H.L., Eguienta Y., Hieu T.T., 2004. A participatory simulation to facilitate farmers' adoption of livestock feeding systems based on conservation agriculture in the uplands of Northern Vietnam. Int. J. Agric. Sustain., 2 (2): 118-132, doi: 10.1080/14735903.2004.9684572

Mikolasek O., Trinh D.K., Medoc J.-M., Porphyre V., 2009. L'intensification écologique d'un modèle de pisciculture intégrée: recycler les effluents d'élevages porcins de la province de Thai Binh (Nord Vietnam). Cah. Agric., 18 (2-3) : 235-241, doi : 10.1684/agr.2009.0295

Molénat M.M., Tran The Thong, 1991. La production porcine au Viet Nam et son amélioration. FAO, Rome, Italie $\left(\mathrm{n}^{\circ} 68\right)$

Moraine M., 2015. Conception et évaluation de systèmes de production intégrant culture et élevage à l'échelle du territoire. Thèse Doct., Université de Toulouse, France, 200 p.

Naylor R., Steinfeld H., Falcon W., Galloway J., Smil V., Bradford E., Alder J., Mooney H., 2005. Losing the links between livestock and land. Science, 310 (5754): 1621-1622, doi: 10.1126/science. 1117856

Nguyen Manh Dzung, 2014. Pig production and marketing in Vietnam. In: Int. Symp. Recent progress in swine breeding and raising technologies, Taiwan, Taiwan, 3-4 June 2014, 145-152

\section{Summary}

Cesaro J.-D., Porphyre V., Duteurtre G. Influence of the industrialization of pig farming on the diversification of mixed livestock integration in Vietnam

Since the early 1990s, pig farming systems in Vietnam have evolved toward more intensive systems, which have been in the process of industrialization since the 2010s. Farmers are increasingly using technologies and services from the agribusiness. This transformation of pork workshops makes it possible to increase meat production but it questions the traditional integration of crop and livestock within farms. Through a historical and geographical approach, this article shows that, even in a process of industrialization of the pig sector, mixed livestock production continues. In 2011, more than $90 \%$ of pig farms included at least one complementary agricultural activity. However, the transformation of livestock systems is leading to a diversification of forms of integration. The new systems tend to organize themselves around the management of effluents to develop agricultural workshops with higher added value, or on the economic complementarity between which risks to take in the face of market uncertainty, at the expense of fodder or livestock feed production on the farm.

Keywords: swine, mixed farming, intensification, agricultural development, Viet Nam
Nguyen Tien Dinh, 2014. Transition alimentaire et essor économique : portrait en régions de la consommation de viandes au Vietnam. Econ. Soc., 10 (36) : 1559-1578

Nguyen Thi Duong Nga, Ho Ngoc Ninh, Pham Van Hung, Lapar M.L., 2014. Smallholder pig value chain development in Vietnam: Situation analysis and trends. ILRI, Nairobi, Kenya

Otsuka K., Liu Y., Yamauchi F., 2016. Growing advantage of large farms in Asia and its implications for global food security. Glob. Food Secur., 11: 5-10, doi: 10.1016/j.gfs.2016.03.001

Pingali P.L., 1997. From subsistence to commercial production systems: The transformation of Asian agriculture. Am. J. Agric. Econ., 79 (2): 628-634, doi: $10.2307 / 1244162$

Porphyre V., Nguyen Que Coi, 2006. The E3P diagnostic project: an introduction about sustainable pig production in Vietnam. In: Pig production development, animal-waste management and environment protection: a case study in Thai Binh province, Northern Vietnam (Eds. Porphyre V., Nguyen Que Coi). CIRAD, Montpellier, France, 10-14

Robinson T., Thornton P.K., Franceschini G., Kruska R.L., Chiozza F., Notenbaert A., Cecchi G., Herrero M., et al., 2011. Global livestock production systems. FAO, Rome, Italy, $152 \mathrm{p}$.

Seré C., Steinfeld H., 1995. World livestock production systems: Current status, issues and trends. FAO, Rome, Italy, $58 \mathrm{p}$.

Smith G., 2017. Vietnam: Grain and Feed Annual 2017. USDA, Foreign Agricultural Service, Hanoi, Vietnam, 29 p. https://gain.fas.usda.gov/ Recent\%20GAIN\%20Publications/Grain\%20and\%20Feed\%20Annual_ Hanoi_Vietnam_4-13-2017.pdf (accessed 3 Apr. 2018)

Tran The Tong, 1973. Pig breeding. In: Agronomical data. Xunhasaba, Hanoi, Vietnam, 128-141

Van Thi Khanh Vu, Tien Tran Minh et Son Thi Thanh Dang, 2007. A survey of manure management on pig farms in Northern Vietnam. Livest. Sci., 112 (3): 288-297

Vu Trong Binh, 2002. La qualité des porcs dans le Nord du Vietnam (delta du Fleuve Rouge) entre organisation des producteurs et évolution des systèmes de production. Thèse Doct., INA-PG, Paris, France, 297 p.

World Bank, 2016. Vietnam 2035: Toward prosperity, creativity, equity, and democracy. World Bank, Washington, USA, 368 p.

\section{Resumen}

Cesaro J.-D., Porphyre V., Duteurtre G. Influencia de la industrialización de las fincas de cerdos sobre la diversificación de la integración de ganado mixto en Vietnam

Desde principios de 1990, los sistemas de fincas de cerdos en Vietnam han evolucionado hacia sistemas más intensivos, los cuáles han estado en proceso de industrialización desde 2010. Los finqueros usan cada vez más tecnología y servicios del sector agro comercial. Esta transformación de talleres de cerdo hace posible el aumento de la producción de carne, pero cuestiona la integración tradicional de una mezcla de ganado en las fincas. A través de un enfoque histórico y geográfico, el presente artículo muestra que, incluso durante un proceso de industrialización del sector porcino, la producción mixta de ganado continúa. En 2011, más de 90\% de las fincas de cerdos incluían al menos otra actividad agrícola complementaria. Sin embargo, la transformación de los sistemas ganaderos está llevando hacia una diversificación de las formas de integración. Los nuevos sistemas tienden a organizarse alrededor del manejo de efluentes para desarrollar talleres agrícolas con mayor valor agregado, o sobre la complementariedad económica entre cuáles riesgos tomar frente a la incertidumbre del mercado, a costa de producción de piensos o de ganado en las fincas.

Palabras calve: cerdo, explotación agrícola combinada, intensificación, desarrollo agrícola, Vietnam 
